\title{
Recent advancements to enhance the therapeutic efficacy of antiepileptic drugs
}

FUQUAN LI ${ }^{1}$

AKHILESH VIKRAM SINGH ${ }^{2, *}$

${ }^{1}$ Zhangqiu District Maternal and Child Health Hospital, Jinan City, Shandong Province, China

${ }^{2}$ School of Epidemiology and Public Health, Datta Meghe Institute of Medical Sciences, Wardha, Maharashtra, India

Accepted November 16, 2020

Published online December 9, 2020

\begin{abstract}
Epilepsy is a multifactorial neurological disorder characterized by recurrent or unprovoked seizures. Over the past two decades, many new antiepileptic drugs (AEDs) were developed and are in use for the treatment of epilepsy. However, drug resistance, drug-drug interaction and adverse events are common problems associated with AEDs. Antiepileptic drugs must be used only if the ratio of efficacy, safety, and tolerability of treatment are favorable and outweigh the disadvantages including treatment costs. The application of novel drug delivery techniques could enhance the efficacy and reduce the toxicity of AEDs. These novel techniques aim to deliver an optimal concentration of the drug more specifically to the seizure focus or foci in the CNS without numerous side-effects. The purpose of this article is to review the recent advancements in antiepileptic treatment and summarize the novel modalities in the route of administration and drug delivery, including gene therapy, for effective treatment of epilepsy.
\end{abstract}

Keywords: antiepileptic drugs, drug delivery, epilepsy, gene therapy, liposomes, nanoparticles

\section{INTRODUCTION}

Epilepsy is a chronic neurological disorder that affects people of all ages and races with a high incidence in elderly people and children. According to the WHO factsheet 2019 (1), about 50 million people worldwide have epilepsy. The pooled incidence rate of epilepsy was 61.4 per 100,000 persons per year, and its incidence was even higher in low/ middle-income countries ( $\sim 80 \%$ ) than in high-income countries (2). Epilepsy is characterized by repeated seizures resulting from recurrent, abnormal, excessive synchronous discharges of cerebral neurons. Epilepsy can be caused by stroke, genetic disorder, brain tumor or infection, and brain injury. There is no clinical confirmation, however, that epilepsy patients infected with COVID-19 or any other infectious disease may have a fever which may trigger seizures (3). Antiepileptic drug (AED) therapy is the mainstay of treatment for most patients, but in some cases, brain surgery may be required. Approximately $70 \%$ of cases of epileptic seizures are controlled with AEDs, albeit with some side-effects (4). The International Board against Epilepsy (ILAE) and the International Bureau of

\footnotetext{
*Correspondence; e-mail: akhileshvikram@gmail.com
} 
Epilepsy (IBE) defined epileptic seizures as a transient occurrence of signs and/or symptoms due to abnormal excessive or synchronous neuronal activity in the brain (5). The main factors involved in the pathophysiology of seizures are: (i) abnormalities in the hippocampus region of the brain, (ii) imbalance in the excitatory and inhibitory neuronal systems along with the generation of a burst discharge from a neuron, and (iii) impairment of gamma-aminobutyric acid (GABA) inhibitory system (6). Based on seizure origin, epilepsy is classified into two types: (i) partial (focal) seizures and (ii) generalized seizures. The diagnosis of epilepsy is generally based on the results of electro-encephalography (EEG), high-resolution cerebral magnetic resonance imaging (MRI) and cerebrospinal fluid (CSF) analysis. The choice of treatment mainly depends on an accurate diagnosis of epilepsy syndrome based on careful patient history and findings by EEG and MRI (7). Over the past few years, many new AEDs have been developed and are in use for antiepileptic treatment $(8,9)$. Treatment options have increased dramatically since the 1990 s with the introduction of new generation AEDs such as vigabatrin, felbamate, gabapentin, lamotrigine, tiagabine, topiramate, levetiracetam, oxcarbazepine, zonisamide, pregabalin, rufinamide, and lacosamide. The newer generations of drugs are more efficacious demonstrating better safety and tolerability profiles compared with the older generation drugs such as phenobarbital, phenytoin, carbamazepine and valproate. The mechanism of antiepileptic drugs acting is depicted in Fig. 1 and categorized in Table I.

However, despite the availability of several new AEDs in the market, many patients still have seizures either because they do not respond to these medications or have related side-effects that prevent continued use of these medications. Even in patients in whom antiepileptic pharmacotherapy is efficacious, current AEDs do not affect the progression of epilepsy. Furthermore, there is currently no AED available which prevents the development of epilepsy.

Adherence to medication forms an important part of the patient's care and is essential for reaching clinical goals. Initiation and termination of the treatment and adherence

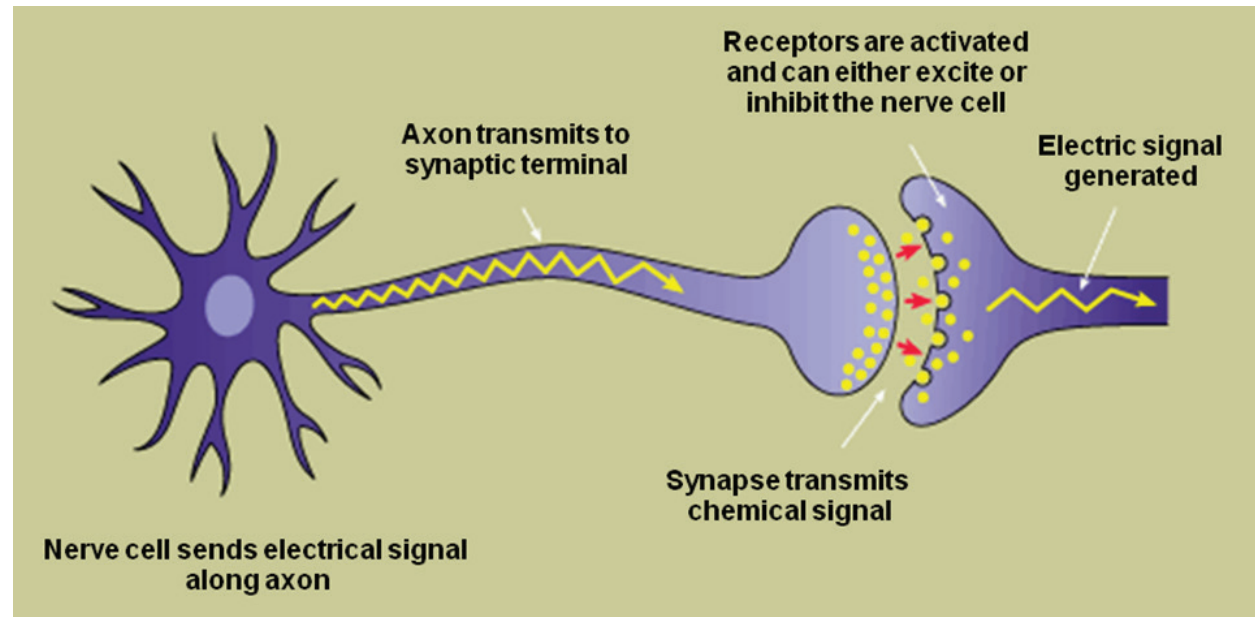

Fig. 1. The mechanism of action of antiepileptic drugs. Taken from resources at The Royal Children's Hospital, Melbourne, Australia (www.rch.org.au), with permission. 


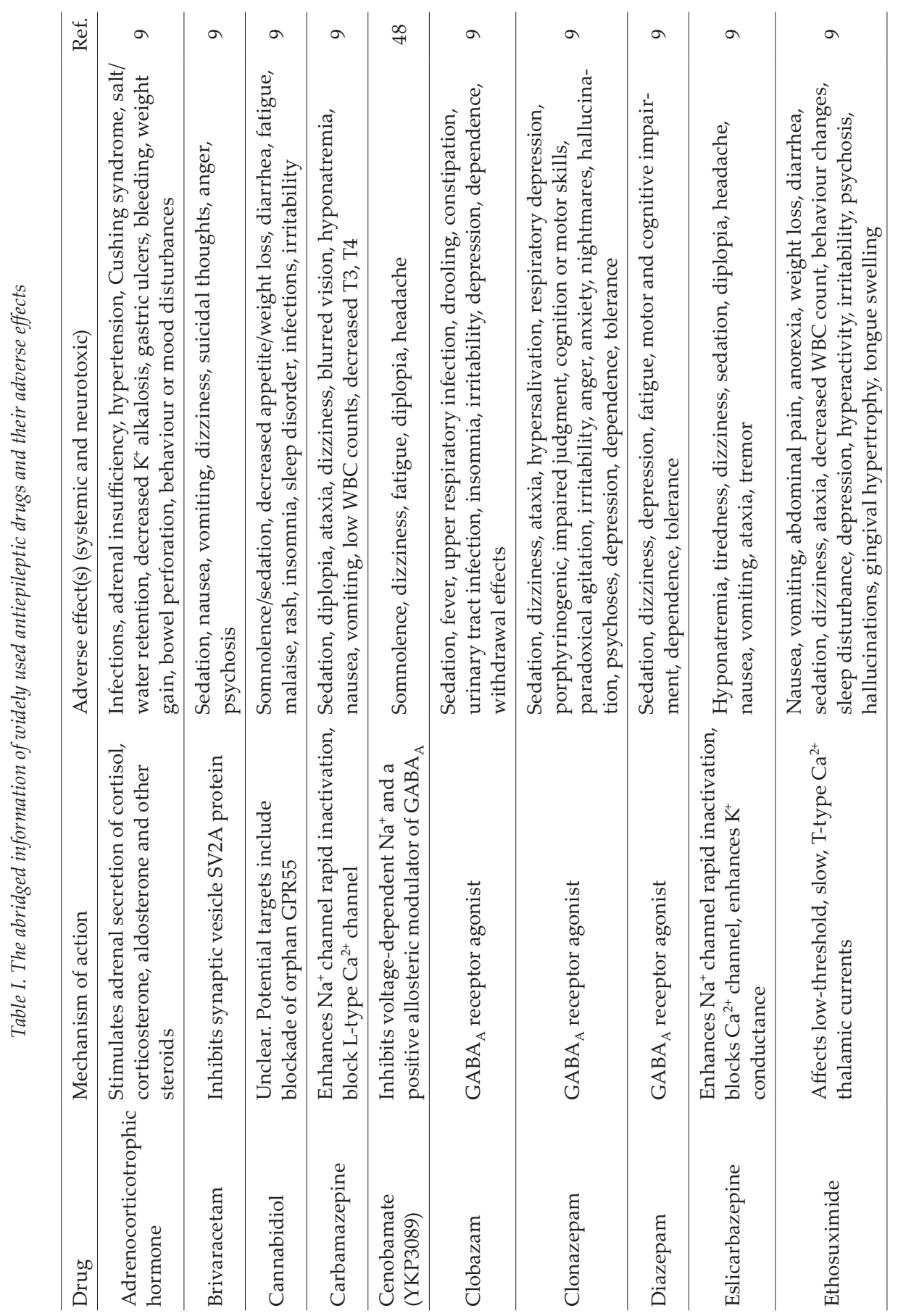




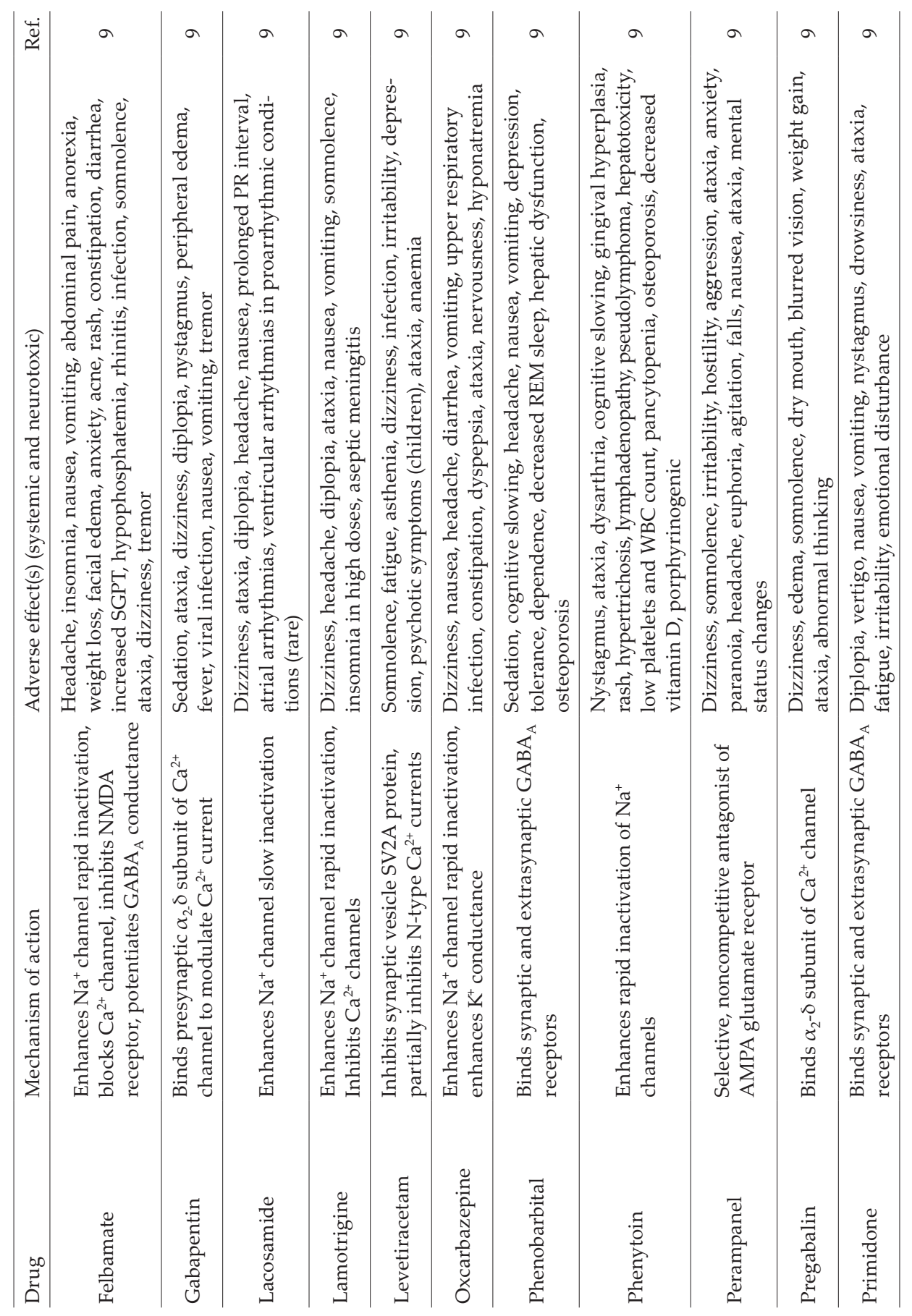




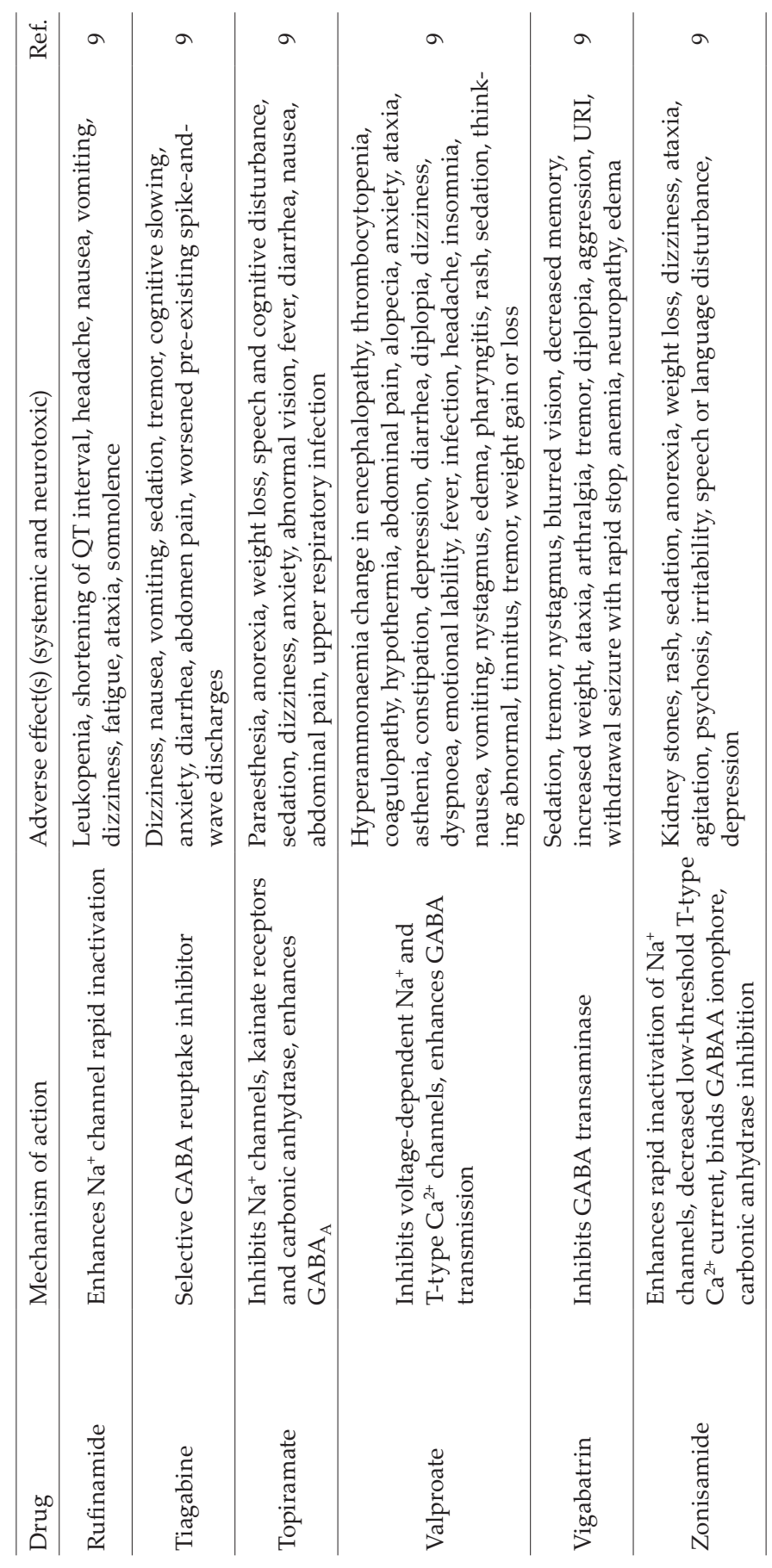


assessments are important tools for epileptic disease management. Therefore, it is important to evaluate any patient comorbidity, especially psychiatric comorbidities, before and after choosing AEDs (8). Currently, there is no proper evidence that could prove that current AEDs could prevent or reduce drug resistance in patients (10). The fact that epilepsy is heterogeneous with enormous variations in etiology and clinical features makes it unlikely that a single drug could eradicate refractory epilepsy.

Given the high cost of clinical drug development, research organizations are emphasizing the development of novel drug delivery methods that could enhance efficacy and reduce the toxicity of AEDs (11). One of the major challenges in drug delivery to the brain is the presence of a blood-brain barrier (BBB) which is the major route for the uptake of different substances such as serum ligands (12). The relative impermeability of the BBB is due to the presence of tight junctions that form impermeable seals between capillary endothelial cells. Recently, many new strategies have been developed to increase the penetration of AEDs in the brain parenchyma.

In this review, we discuss the recent developments in the field of drug delivery for the treatment of epilepsy. We are also reviewing new therapeutic compounds that are in the clinical pipeline, or currently in the clinical evaluation stage for the cure of epilepsy and its symptoms. The recent advancement in antiepileptic treatment can be divided into four categories: (i) new antiepileptic drugs, (ii) novel delivery approaches, (iii) novel route approaches, $(i v)$ gene therapy.

\section{CURRENT ANTIEPILEPTIC DRUGS}

Paraldehyde was the first AED that was introduced in 1882, and since then many new drugs have been developed to reduce seizure frequency and to achieve seizure freedom in patients. Currently available antiepileptic drugs are listed in Table I, and few newer drugs which are presently under clinical development phases are listed in Table II. The choice of AED depends on several factors such as seizure type, presence of an epilepsy syndrome, other medications, comorbidities, lifestyle, and patient preference. As per the literature, antiepileptic monotherapy should start when the initial treatment fails to control seizures. Subsequent management should be undertaken when the initial monotherapy also fails to produce the desired action (13). Carbamazepine is the drug of choice for focal onset seizures, while divalproex sodium is used for generalized seizures.

\section{NOVEL DELIVERY APPROACHES}

Theoretically, direct delivery of AEDs to the CNS has a higher therapeutic-toxic ratio than the systemic drugs. However, several possible methods have been established for the direct delivery of AEDs to the CNS $(14,15)$.

\section{Convection-enhanced delivery (CED)}

CED facilitates the targeted delivery of drugs to the brain (16). CED is a promising technique that uses positive hydrostatic pressure to deliver therapeutics into the interstitial space within a localized region of the brain parenchyma. CED circumvents the BBB and provides a homogenous distribution than bolus deposition (focal injection) or any 


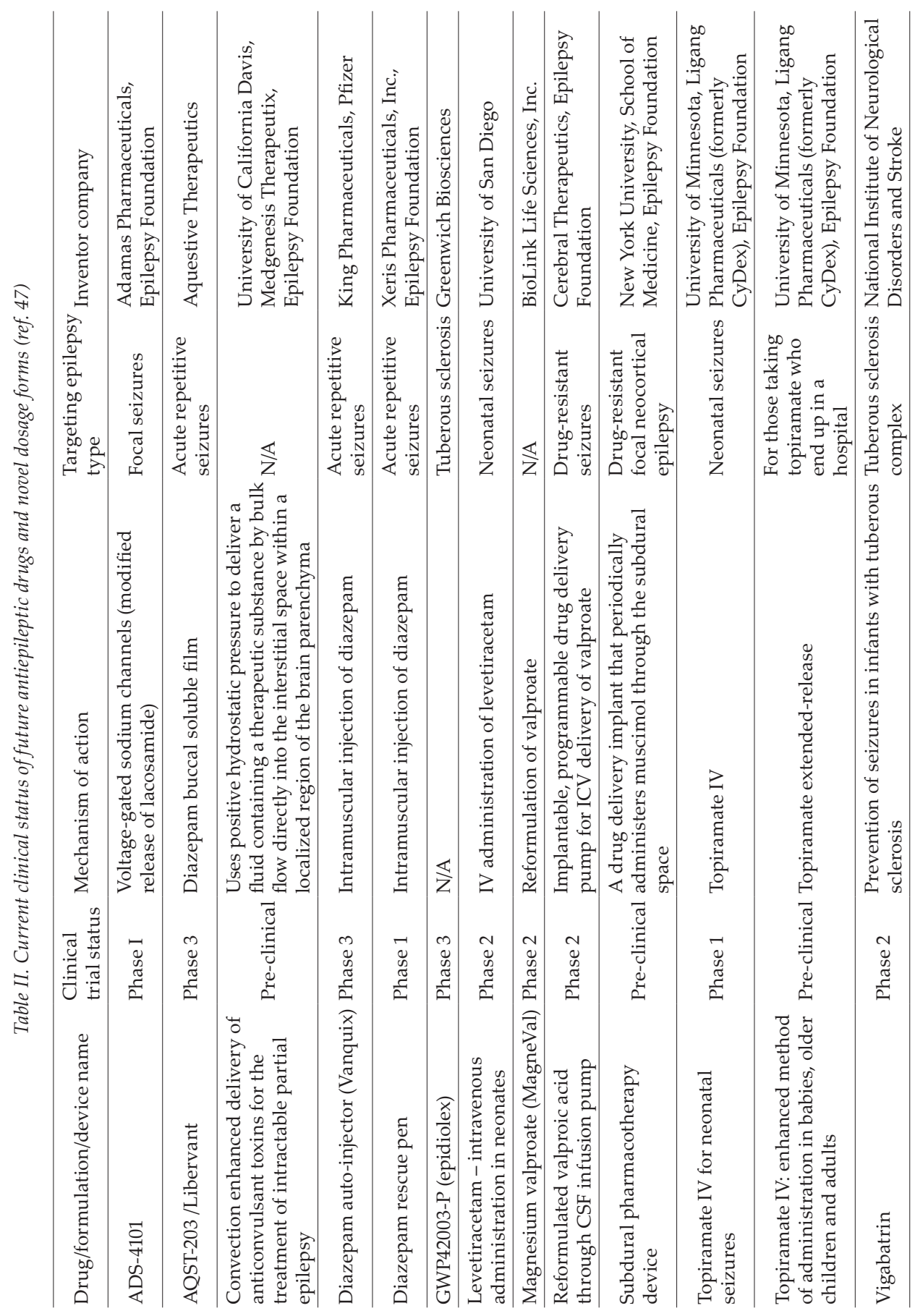


other diffusion-based delivery approach. CED avoids the systemic toxicities of orally administered AEDs and bystander effects on non-epileptic brain regions. In a typical CED application, the drug is dissolved in a saline solution and is delivered by slow infusion under positive pressure through a fine cannula $(17,18)$.

\section{Drug delivery by liposome}

Liposomes are microscopic spherical vesicles consisting of a phospholipid bilayer that is used to encapsulate chemical compounds. In this form, the vehicle is able to release the drug in a steady manner over a long period of time. The liposome can bind to a specific receptor in the region of interest and the drug entrapped in the liposome is delivered into the brain where seizures were generated. Difficulty in drug penetration across the BBB is one of the major limitations of liposomes (19).

\section{Nanoparticles}

Nanoparticle drug delivery systems use nanoparticles for the targeted delivery and controlled release of therapeutic agents. Nanoparticles could also be used for AEDs delivery to the brain, in which the drug is encapsulated within a nanoscale delivery system. The nanoparticles are composed of biodegradable polymers with a size ranging from 10 to $1000 \mathrm{~nm}$. Examples of polymers used in nanoparticle formulations include poly(lactic-co-glycolic acid) (PLGA), which is used more frequently due to its biocompatibility and safety. Some researchers reported that the degradation property of poly(lactic-co-glycolic) acid can be controlled by changing the ratio of the copolymer $(20,21)$. The therapeutic efficacy of ethosuximide-loaded nanogel in a rat model using the depot drug delivery technique showed a promising alternative to available ethosuximide formulation (22).

By using the bio-conjugation technique, polymers can be conjugated to the liposomal surface and nanoparticles to increase its biological half-life in the systemic circulation. These polymer chains form a 'stealth' like structure, thus the particles are no longer opsonized and increase the circulation time. Examples of such polymers are polyvinyl alcohol, polysaccharides, polyethylene glycol (PEG), and poly-acrylamide. To produce stealth-like particles, PEG is preferred due to its hydrophilic nature. PEG attaches to the colloidal carrier surface by adsorption, physical entrapment, and/or covalent attachment and protects the plasma protein from the opsonization process. However, it is uncertain whether PEG, which can increase the circulation time of AEDs after intravenous injection, can also cross the BBB. The PEG-based nanoparticle may be conjugated with a targeting moiety (cellpenetrating peptides) to facilitate the passage of AEDs across the BBB.

\section{Efflux pumps}

With the addition of some biochemical substances to the drug delivery system, the permeability of the drug across BBB can be increased. Examples of such compounds include multidrug resistance-associated proteins (MRPs), P-glycoprotein (PgP) and breast cancer resistance proteins (BCRP). These proteins belong to the superfamily of ATP binding cassette $(\mathrm{ABC})$ transporter, expressed in the neurons, endothelial cells, and epileptogenic brain tissue in patients with intractable epilepsy. It hydrolyzes ATP to transfer mole- 
cules into the systemic circulation against their concentration gradient, and by doing this affects drug localization into the CNS.

The literature showed that anticonvulsant treatments can be improved by blocking the $\mathrm{ABC}$ transporter system in the brain. For example, verapamil (calcium channel blocker) inhibits the pumping action of $\mathrm{PgP}$, thus a greater control in seizure is experienced by patients who use verapamil and AEDs. The disadvantage of this method includes the variable ability of efflux pumps to transport the substrate from species to species and all the AEDs are not proper substrates for PgP, MRPs, and BCRP. More investigations are undertaken to understand the particular mechanism of efflux pumps on AEDs (23).

\section{Drug wafer}

A drug wafer consists of an interwoven AED in a polymer matrix. In these formulations, the polymers are dissolved with the drug and it slowly releases the medication for a month or years. Nowadays, neurologists and neurosurgeons use the drug wafer such as BCNU-containing gliadel wafer also for tumor resection. The efficacy of phenytoin was investigated by Tamargo et al. (24) and to prove the same, they used a cobalt-induced rat model for epilepsy and phenytoin as a drug wafer which was administered through the intra-cerebral route. The drug (phenytoin) was slowly released up to a period of 4 months; as a result, there was progress in the behavioral seizure scale in comparison to animals who received drugs without drug wafer. The main drawback of drug wafers includes low potencies, low solubility, and the drug-releasing capacity was also nonlinear which could provide a narrow therapeutic ratio.

\section{Perfusion by catheters}

In this method, a catheter and a pump are used to deliver an AED. The catheter is attached to a pump to infuse the drug at a constant rate. Eder et al. (25) designed a rat model to study this drug delivery system. In order to evaluate this system, a small bone window was designed in the left parietal region of the rat, and an AED (bicuculline methiodide) was administered which generated an epileptic form spike.

\section{Pro-drugs}

The pro-drug (inactive form) is converted into an active form of the drug and a nontoxic moiety after biotransformation. The pro-drugs are attached to a distinct compound that is removed via hydrolysis (in vivo) or enzymatic cleavage. The pro-drug can cross the BBB because of the attached moiety, thereby making the pro-drug more lipophilic. A prodrug of valproic acid, named DP-VPA (DP16) is prepared by linking VPA and lecithin (a phospholipid), which gets activated in the systemic circulation. The active drug (valproic acid) is released by phospholipase A2 following the cleavage of lecithin. Using this method, the valproic acid becomes overactive and can reach the target site more efficiently compared with plain valproic acid. Systemic toxicity is greatly reduced by limiting the activation of pro-drug to the seizure focus. After cessation of seizure, the over activation of pro-drug is prevented, since the neurons exit their excited state and the enzymatic activity is decreased (26). 


\section{Hyperosmolar BBB opening}

The osmolarity of the blood flowing through the capillary of the brain can be increased by altering the function of the BBB. A hyperosmolar solution such as mannitol $(25 \%)$ when administered intra-arterially reduces the size of the brain capillary (endothelial cells). Thus, the permeability of the BBB for AEDs may be increased by increasing the width of the tight junction between them. The hyper-osmotic effect on BBB opening is spontaneously reversible and acts for a short period of time. After the addition of mannitol, the permeability of the drug to the BBB gets enhanced and returns to its normal range after $8 \mathrm{~h}$. The common side-effects of this method include edema and toxicity (27).

\section{Monolithic devices}

Monolithic devices are produced by different processes such as casting, melt extrusion and electrospinning method (28). These devices are applied to the brain surface or the brain parenchyma as pellets, rods, disks, and fibers.

\section{NOVEL ROUTE APPROACHES}

The development of medical devices opened new hope for the increased therapeutic efficacy of antiepileptic drugs through neuromodulation or acting directly (Table III).

\section{Non-oral routes}

Nasal/rectal routes. - The nasal mucosa can be used for non-invasive systemic administration of drugs. Water-soluble drugs such as benzodiazepine, midazolam are administered through the intranasal route. Clinical studies have proven that the intranasal administration of midazolam exerts its effects slowly compared with intravenous administration of diazepam in children (29). However, administration of AEDs through the nasal route has some major limitations as respiration becomes shallow and irregular during seizures. Recently, USFDA (30) has approved the rectal route for drug delivery application. In a comparative study of route preference among patients with epilepsy, midazolam administered through nasal route was equally efficacious to diazepam that was administered through the rectal route for the suppression of seizure exacerbations (31).

Trans-dermal route. - One of the most common routes for drug administration is via the dermal and epidermal layers of the skin. Antiepileptic drugs such as lidocaine are administered by the intradermal route. However, several clinical studies have demonstrated that lidocaine administration through the skin caused rapid remission of recurrent seizures $(32,33)$.

\section{Localized routes}

Intracerebroventricular administration. - In this technique, the drug is administered directly into the cerebrospinal fluid (CSF) by an intracerebroventricular route in which an outlet catheter leading from an implantable reservoir/pump is used. Thus, problems 


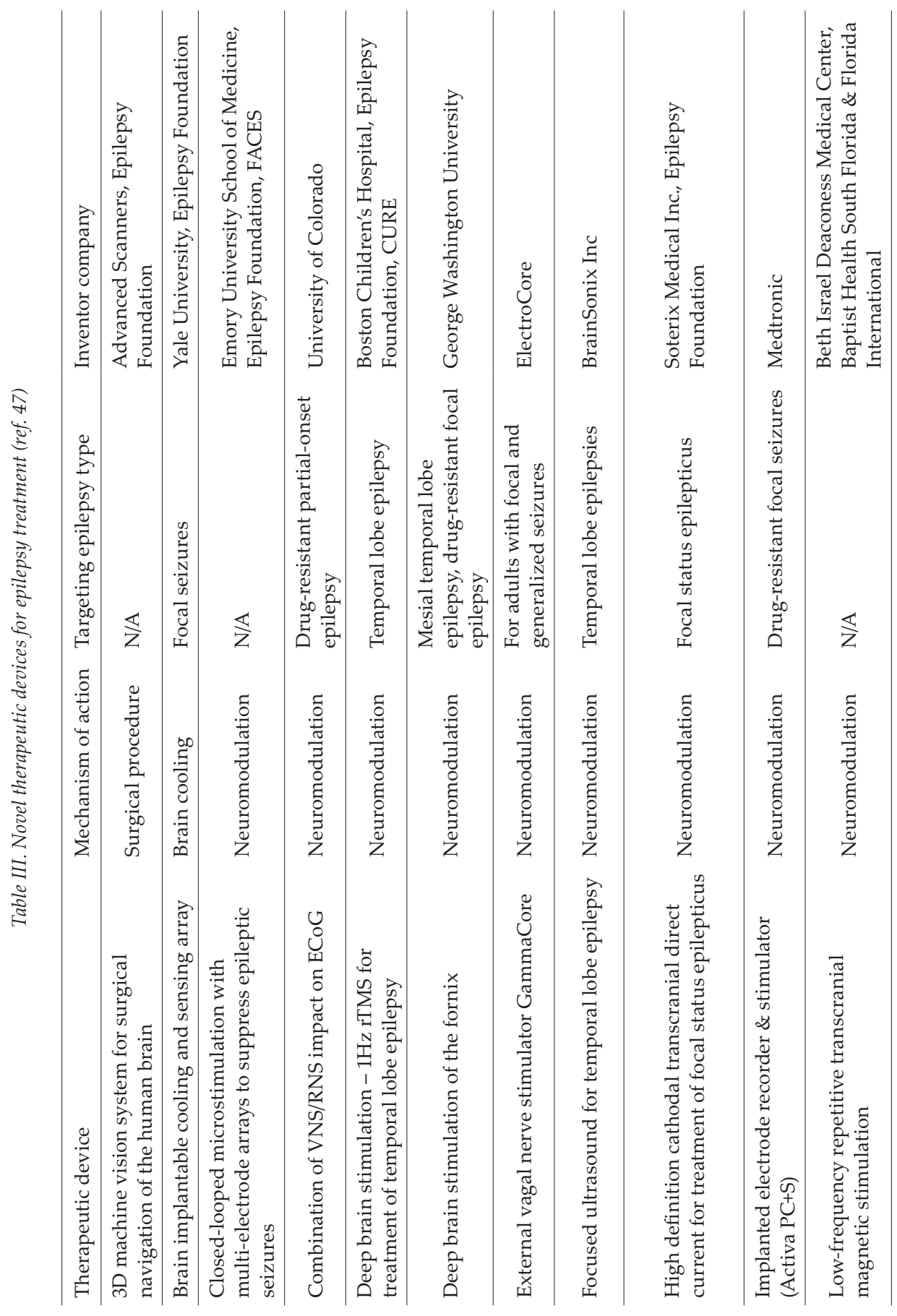




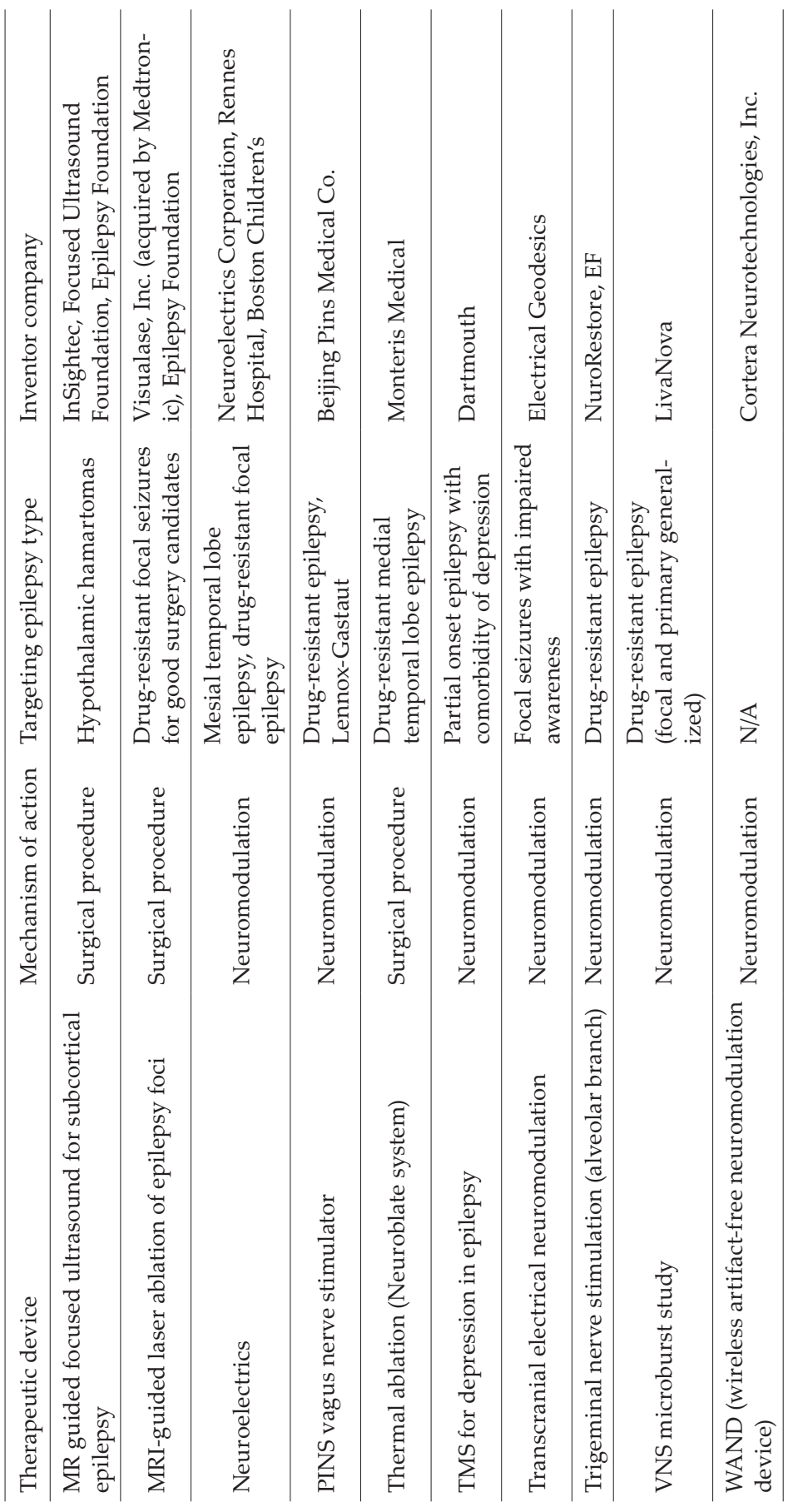


associated with intravenous administration such as opsonization, drug metabolism, systemic toxicity can be reduced. The limitations of this technique include restricted drug penetration into the brain parenchyma. Intracerebroventricular administration is useful for the local deposition of the drug at the ependymal surface of the brain, but not for drug delivery to cells that are far from the ependymal surface. The movement of drug molecules from the CSF to the brain parenchyma (by diffusion) is very low because of high tortuosity that restricted the pore size of the extracellular space $(34,35)$.

Intracerebral administration. - Alternative method for AEDs administration in the ventricle is the delivery of the drug to the parenchyma of the brain by an implant/injection. Studies were carried out on drugs such as adenosine, which was injected into the seizure focus/ventricle. The antiepileptic effect of these delivery strategies after seizure onset by an intra-cerebral penicillin injection has been investigated. The adenosine reduces the mean spike frequency, but not the amplitude. Local adenosine injection to the frontal area showed a reduction in the mean spike frequency and amplitude. The antiepileptic effect increased after drug administration, suggesting that the adenosine injection given directly to a seizure focus was more effective than intracerebroventricular administration (36).

Intraventricular/intrathecal route. - Drugs administered into CSF through the intraventricular/intrathecal route are found to be safer compared with other routes. This route does not cause any surgical trauma to the brain and is considered safer compared to available techniques involving surgical procedures. Factors affecting CSF drug delivery include penetration rate and drug concentration in the brain tissue. In order to prevent seizures in epilepsy, the movement of medication (intrathecal/intraventricular route) must be in bulk convection and should penetrate faster under pressure (37). A preclinical study of AEDs administered through the intrathecal route in animal models produces a severe decrease in motor activity. Intrathecal administration of baclofen was evaluated in the reduction of seizure episodes in the pediatric population. About $13.3 \%$ of pediatric patients who received baclofen via the intrathecal route showed a decrease in seizure frequency $(38,39)$.

\section{GENE THERAPY}

Studies have shown that gene therapy can be used for the treatment of acquired and genetic diseases. Gene therapy can also be used to treat diseases such as cancer, hemophilia, AIDS, neurodegenerative disorders. Preclinical studies have shown that gene therapy is capable of suppressing seizures at their site of origin $(40,41)$.

The most common method of gene delivery to the brain involves the packaging of the molecular apparatus of gene expression into a deactivated viral vector, followed by direct focal microinjection into the relevant brain region $(42,43)$.

The insertion of genetic code and genetic expression depends on the ability of viruses to invade host cells. Viruses such as recombinant adeno-associated viruses (rAAVs) are used, which have low immunogenicity, prolonged-expression, and tropism for post-mitotic neurons. However, there could be some limitations with the selected gene for gene therapy approach in epilepsy for preclinical studies. The investigation started with the inhibitory neurotransmission activity mediated by several components like GABA, subunits of GABAa receptor and transduction of glutamic acid decarboxylase. 
In epilepsy, the GABA receptors are down-regulated and in some cases, loss of GABA-ergic neurons causes chronic epilepsy. To overcome this condition, some endogenous neuromodulators like galanin, neuropeptide Y (NPY) have been used because of their anticonvulsant activity. These neuromodulators exert/produce their inhibitory activity on the presynaptic glutamate release and by gene transduction process the genetic code is inserted/entrapped into host neurons which results in over-expression of peptide release to enhance their neuroprotective activity. Gene therapy has been attempted to produce antiepileptogenic, antiseizure and disease-modifying effects (44).

According to Sills (45), NPY-based gene therapy provides some potential for antiseizure and antiepileptogenic effects. The author used an experimental model that consists of temporal lobe epilepsy with a short period of acute status epilepticus, induced in the hippocampus by electrical stimulation. After a few weeks, a 24-h video of EEG monitoring was recorded with an unprovoked recurrent seizure. Afterward, the NPY gene therapy was started with the randomized animal in which a control vector (without NPY gene) and the rAAV vector were given directly by microinjection (bilateral) into the hippocampus. Then, the rat/animal was evaluated for seizure activity by using a 24-h video of EEG after 4 weeks. During this time the neuronal transduction and overexpression of the NPY gene were generated. On the other hand, the control animals developed some epilepsy with spontaneous seizures. The animals which were treated with NPY gene therapy showed a significant reduction in seizure activity. The overexpression of the NPY gene was limited/ restricted to some regions of the hippocampus, that is, the dentate gyrus. The immunohistochemical studies revealed that the overexpression mainly depends on the antiepileptogenic activity. In the immunohistochemical method, the tissue was harvested by an ex vivo process, using video-EEG monitoring.

In gene therapy, safety is the main concern for an epileptic patient. By reverse recombination or mutagenesis process, the viral vector might be deactivated which is the main safety concern in gene therapy. In the long term, gene expression shows unexpected adverse effects. A focal delivery through stereotaxic neurosurgery creates a barrier for the use of gene therapy in refractory epilepsy and those patients are unsuitable for surgery. Gene therapy can be applied to surgically remediable lesions to reject the injected part/ organ. However, in complex epilepsy, single-gene transduction is not enough to produce remarkable action.

\section{FUTURE RESEARCH CHALLENGES}

Several AEDs with superior efficacy are still under research and development. Many patients experience memory disorders and severe adverse effects with the use of available AEDs. In children, a number of epilepsy syndromes remain constant due to drug resistance. The new antiepileptic drug discovery programs have relied primarily on a screening process by using traditional animal models that act primarily on ion channels or neurotransmitter receptors. The new AEDs are discovered when new chemical entities were subjected to systemic testing and found by chance to have antiepileptic properties. Some new molecules were rationally designed in order to enhance their efficacy based on currently available agents to eliminate side-effects.

Apart from this, researchers need to define new targets for developing a better drug. For this purpose, the neurobiology of the epileptogenic region in the brain and the mech- 
anisms that lead to the emergence of epileptic seizures must be thoroughly investigated $(46,47)$. The required technological parameters for identifying new drug targets can be done by:

- developing the molecular and cellular analysis of tissue specimens,

- gene transfer technology,

- generation of genetically modified animals, and

- advanced imaging and electrophysiological techniques.

\section{CONCLUSIONS}

Conventionally, antiepileptic drugs are administered by oral and intravenous routes. However, due to metabolic, physiological, and genetic polymorphism related limitations, an individualized treatment approach should be adopted for optimal management of epilepsy. For epilepsy patients that do not respond well to monotherapy, a combination therapy must be considered; however, overtreatment should be carefully avoided due to systemic side-effects and neurotoxicity. Novel treatments and delivery approaches aimed at either disrupting or by-passing the blood-brain barrier opened a new era in the treatment of epilepsy. Research on human genetics also created a glimmer in this therapeutic domain for a more specialized approach to epilepsy treatment. Healthcare professionals and patients place great hopes for better efficacy and tolerability of new antiepileptic drugs and delivery approaches in different clinical trials.

Acknowledgements. - Akhilesh Vikram Singh was affiliated with the Department of Pharmaceutical Sciences, Dibrugarh University, Assam, India, at the time of manuscript development.

Abbreviations, acronyms, symbols. - ABC - ATP binding cassette, AED - anti-epileptic drugs, AMPA - $\alpha$-amino-3-hydroxy-5-methyl-4-isoxazolepropionic acid, BBB - blood-brain barrier, BCRP - breast cancer resistance proteins, CED - convection-enhanced delivery, CNS - central nervous system, CSF - cerebrospinal fluid, EEG - electroencephalography, FACES - finding a cure for epilepsy and seizures, GABA - gamma-aminobutyric acid, GPR- G-protein coupled receptor, IBE - International Bureau of Epilepsy, ILAE - International Board against Epilepsy, MRI - magnetic resonance imaging, MRPs - multidrug resistance-associated proteins, NINDS - National Institute of Neurological Disorders and Stroke, NMDA - N-methyl-D-aspartate receptor, NPY - neuropeptide Y, PEG - polyethylene glycol, PgP - P-glycoprotein, PLGA - poly(lactic-co-glycolic) acid, rAAVs - recombinant adeno-associated viruses, rTMS - repetitive transcranial magnetic stimulation, SV2A - synaptic vesicle glycoprotein 2A, VPA - valproic acid, WAND - wireless artifact-free neuromodulation device.

\section{REFERENCES}

1. World Health Organization, Epilepsy Fact Sheet, WHO, June 2019, Geneve; https://www.who.int/ news-room/fact-sheets/detail/epilepsy; last access date October 28, 2020.

2. E. Beghi, The epidemiology of epilepsy, Neuroepidemiology 54 (2020) 185-191; https://doi.org/ $10.1159 / 000503831$

3. N. Kuroda, Epilepsy and COVID-19: Associations and important considerations, Epilepsy Behav. 108 (2020) Article ID 107122 (3 pages); https://doi.org/10.1016/j.yebeh.2020.107122 
4. H. P. Rang, M. M. Dall, J. M. Ritter and R. J. Flower, Antiepileptic Drugs, in Rang and Dale's Pharmacology, Elsevier, Amsterdam 2007, pp. 540-551.

5. R. S. Fisher, W. van Emde Boas, W. Blume, C. Elger, P. Genton, P. Lee and J. Jr. Engel, Epileptic seizures and epilepsy: definitions proposed by the International League Against Epilepsy (ILAE) and the International Bureau for Epilepsy (IBE), Epilepsia 46 (2005) 470-472; https://doi.org/10.1111/ j.0013-9580.2005.66104.x

6. S. Engelborghs, R. D’Hooge and P. P. De Deyn, Pathophysiology of epilepsy, Acta Neurol. Belg. 100 (2000) 201-213.

7. P. Genton, P. Gelisse, P. Thomas and C. Dravet, Do carbamazepine and phenytoin aggravate juvenile myoclonic epilepsy? Neurology 55 (2000) 1106-1109; https://doi.org/10.1212/wnl.55.8.1106

8. S. C. Schachter, Currently available antiepileptic drugs, Neurotherapeutics 4 (2007) 4-11; https://doi. org/10.1016/j.nurt.2006.11.005

9. D. G. Vossler, M. Weingarten and B. E. Gidal, Summary of antiepileptic drugs available in the United States of America, Epilepsy Curr. 18 (2018) 1-26; https://doi.org/10.5698/1535-7597.18.4s1.1

10. E. Perucca and T. Tomson, The pharmacological treatment of epilepsy in adults, Lancet Neurol. 10 (2011) 446-456; https://doi.org/10.1016/S1474-4422(11)70047-3

11. D. Schmidt and W. Loscher, Drug resistance in epilepsy, putative neurobiologic and clinical mechanisms, Epilepsia 46 (2005) 858-877; https://doi.org/10.1111/j.1528-1167.2005.54904.x

12. S. Beyenburg, J. Bauer and M. Reuber, New drugs for the treatment of epilepsy: a practical approach, Postgrad. Med. J. 80 (2004) 581-587; https://doi.org/10.1136/pgmj.2004.019976

13. S. K. Bhattacharya, G. P. Rauniar and B. P. Das, Recent advances in the management of epilepsy: a review, Kathmandu Univ. Med. J. (KUMJ) 3 (2005) 431-437.

14. R. S. Fisher and J. Ho, Potential new methods for antiepileptic drug delivery, CNS Drugs 16 (2002) 579-593; https://doi.org/10.2165/00023210-200216090-00001

15. R. S. Fisher and D. K. Chen, New routes for delivery of anti-epileptic medications, Acta Neurol. Taiwan 15 (2006) 225-231.

16. J. H. Sampson, M. L. Brady, N. A. Petry, D. Croteau, A. H. Friedman, H. S. Friedman, T. Wong, D. D. D. Bigner, I. Pastan, R. K. K. Puri and C. Pedain, Intracerebral infusate distribution by convection-enhanced delivery in humans with malignant gliomas: descriptive effects of target anatomy and catheter positioning, Neurosurgery 60 (2007) ONS-89-ONS-98; https://doi.org/10.1227/01. NEU.0000249256.09289.5F

17. M. A. Rogawski, Convection-enhanced delivery in the treatment of epilepsy, Neurotherapeutics 6 (2009) 344-351; https://doi.org/10.1016/j.nurt.2009.01.017

18. A. Van Dycke, R. Raedt, K. Vonck and P. Boon, Local delivery strategies in epilepsy: a focus on adenosine, Seizure 20 (2011) 376-382; https://doi.org/10.1016/j.seizure.2011.03.003

19. N. Mori, A. Kurokouchi, K. Osonoe, H. Saitoh, K. Ariga, K. Suzuki and Y. Iwata, Liposome-entrapped phenytoin locally suppresses amygdaloid epileptogenic focus created by db-cAMP/ EDTA in rats, Brain Res. 703 (1995) 184-190; https://doi.org/10.1016/0006-8993(95)01095-5

20. M. F. Bennewitz and W. M. Saltzman, Nanotechnology for delivery of drugs to the brain for epilepsy, Neurotherapeutics 6 (2009) 323-336; https://doi.org/10.1016/j.nurt.2009.01.018

21. Y. Malam, E. J. Lim and A. M. Seifalian, Current trends in the application of nanoparticles in drug delivery, Curr. Med. Chem. 18 (2011) 1067-1078; https://doi.org/10.2174/092986711794940860

22. M.-H. Hsiao, M. Larsson, A. Larsson, H. Evenbratt, Y.-Y. Chen, Y.-Y. Chen and D.-M. Liu, Design and characterization of a novel amphiphilic chitosan nanocapsule-based thermo-gelling biogel with sustained in vivo release of the hydrophilic anti-epilepsy drug ethosuximide, J. Control. Release 161 (2012) 942-948; https://doi.org/10.1016/j.jconrel.2012.05.038 
23. M. D. Farwell, D. J. Chong, Y. Iida, S. A. Bae, B. Easwaramoorthy and M. Ichise, Imaging P-glycoprotein function in rats using [(11) C]-N-desmethyl-loperamide, Ann. Nucl. Med. 27 (2013) 618-24; https://doi.org/10.1007/s12149-013-0725-5

24. R. J. Tamargo, L. A. Rossell, E. H. Kossoff, B. M. Tyler, M. G. Ewend and J. J. Aryanpur, The intracerebral administration of phenytoin using controlled-release polymers reduces experimental seizures in rats, Epilepsy Res. 48 (2002) 145-155; https://doi.org/10.1016/s0920-1211(01)00330-8

25. H. G. Eder, D. B. Jones and R. S. Fisher, Local perfusion of diazepam attenuates interictal and ictal events in the bicuculline model of epilepsy in rats, Epilepsia 38 (1997) 516-521; https://doi. org/10.1111/j.1528-1157.1997.tb01134.x

26. M. H. Semreen, A. N. El-Shorbagi, T. H. Al-Tel and I. M. Alsalahat, Targeting $\gamma$-aminobutyric acid (GABA) carriers to the brain: potential relevance as antiepileptic pro-drugs, Med. Chem. 6 (2010) 144-149; https://doi.org/10.2174/1573406411006030144

27. A. H. Ropper, Hyperosmolar therapy for raised intracranial pressure, N. Engl. J. Med. 367 (2012) 746-752; https://doi.org/10.1056/NEJMct1206321

28. A. J. Halliday, S. E. Moulton, G. G. Wallace and M. J. Cook, Novel methods of antiepileptic drug delivery - polymer-based implants, Adv. Drug Deliv. Rev. 64 (2012) 953-964; https://doi.org/10.1016/j. addr.2012.04.004

29. A. Thakker and P. Shanbag, A randomized controlled trial of intranasal-midazolam versus intravenous-diazepam for acute childhood seizures, J. Neurol. 260 (2013) 470-474; https://doi.org/10.1007/ s00415-012-6659-3

30. J. M. Pellock, Safety of diastat, a rectal gel formulation of diazepam for acute seizure treatment, Drug Safety 27 (2004) 383-392; https://doi.org/10.2165/00002018-200427060-00003

31. G. J. de Haan, P. van der Geest, G. Doelman, E. Bertram and P. A. Edelbroek, A comparison of midazolam nasal spray and diazepam rectal solution for the residential treatment of seizure exacerbations, Epilepsia 51 (2010) 478-482; https://doi.org/10.1111/j.1528-1167.2009.02333.x

32. A. Okumura, T. Tanabe, T. Kato, F. Hayakawa and K. Watanabe, A pilot study on lidocaine tape therapy for convulsions with mild gastroenteritis, Brain Dev. 26 (2004) 525-529; https://doi. org/10.1016/j.braindev.2004.02.009

33. K. Mori, H. Ito, Y. Toda, T. Hashimoto, M. Miyazaki, T. Saijo and Y. Kuroda, Successful management of intractable epilepsy with lidocaine tapes and continuous subcutaneous lidocaine infusion, Epilepsia 45 (2004) 1287-1290; https://doi.org/10.1111/j.0013-9580.2004.17304.x

34. C. Harada, T. Hirai, Y. Fujii, S. Harusawa, T. Kurihara and C. Kamei, Intracerebroventricular administration of histamine $\mathrm{H} 3$ receptor antagonists decreases seizures in rat models of epilepsia, Methods Find. Exp. Clin. Pharmacol. 26 (2004) 263-270.

35. J. A. Barcia and J. M. Gallego, Intraventricular and intracerebral delivery of anti-epileptic drugs in the kindling model, Neurotherapeutics 6 (2009) 337-343; https://doi.org/10.1016/j.nurt.2009.01.015

36. M. Yildirim and C. Marangoz, Anticonvulsant effects of focal and intracerebroventricular adenosine on penicillin-induced epileptiform activity in rats, Brain Res. 1127 (2006) 193-200; https:// doi.org/10.1016/j.brainres.2006.10.024

37. A. Misra, S. Ganesh, A. Shahiwala and S. P. Shah, Drug delivery to the central nervous system: a review, J. Pharm. Pharm. Sci. 6 (2003) 252-273.

38. J. M. Gonzàlez-Darder, E. Gómez-Cárdenas, M. Guerrero, D. Segura-Pastor and J. L. Gil-Salú, Intrathecal antiepileptic drugs in experimental epilepsy, Stereotact. Funct. Neurosurg. 57 (1991) 147-155; https://doi.org/10.1159/000099566

39. V. Buonaguro, B. Scelsa, D. Curci, S. Monforte, T. Iuorno and F. Motta, Epilepsy and intrathecal baclofen therapy in children with cerebral palsy, Pediatr. Neurol. 33 (2005) 110-113; https://doi. org/10.1016/j.pediatrneurol.2005.03.002 
40. V. Riban, H. L. Fitzsimons and M. J. During, Gene therapy in epilepsy, Epilepsia 50 (2009) 24-32; https://doi.org/10.1111/j.1528-1167.2008.01743.x

41. M. C. Walker, S. Schorge, D. M. Kullmann, R. C. Wykes, J. H. Heeroma and L. Mantoan, Gene therapy in status epilepticus, Epilepsia 54 (2013) 43-45; https://doi.org/10.1111/epi.12275

42. W. Löscher, M. Gernert and U. Heinemann, Cell and gene therapies in epilepsy - promising avenues or blind alleys? Trends Neurosci. 31 (2008) 62-73; https://doi.org/10.1016/j.tins.2007.11.012

43. A. Mountain, Gene therapy: The first decade, Trends Biotechnol. 18 (2000) 119-128; https://doi. org/10.1016/s0167-7799(99)01416-x

44. M. Simonato, Gene therapy for epilepsy, Epilepsy Behav. 38 (2014) 125-130; https://doi.org/10.1016/j. yebeh.2013.09.013

45. G. J. Sills, Novel therapeutic strategies for epilepsy-releasing the gene Genie, Epilepsy Curr. 9 (2009) 26-27; https://doi.org/10.1111/j.1535-7511.2008.01280.x

46. M. Baulac and A. Pitkänen, Research priorities in epilepsy for the next decade - a representative view of the European scientific community, Epilepsia 50 (2009) 571-578; https://doi. org/10.1111/j.1528-1167.2008.01811.x

47. D. K. Nguyen and S. S. Spencer, Recent advances in the treatment of epilepsy, Arch Neurol. 60 (2003) 929-935; https://doi.org/10.1001/archneur.60.7.929

48. Epilepsy Foundation, Seizure Medication List, Landover (MD), September 2010; https://www.epilepsy.com/learn/treating-seizures-and-epilepsy/seizure-medication-list; last access date October 24,2020

49. Epilepsy Foundation, Epilepsy Pipeline Tracker, Landover (MD), October 2020; https://www.epilepsy.com/pipeline-listing-page; last access date October 22, 2020 